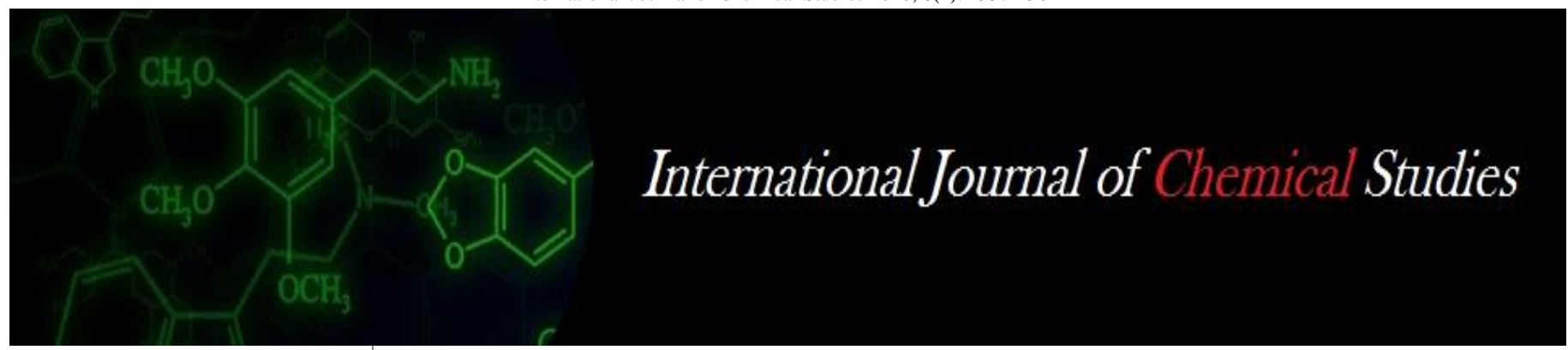

P-ISSN: 2349-8528

E-ISSN: 2321-4902

www.chemijournal.com

IJCS 2020; 8(2): 1957-1962

(C) 2020 IJCS

Received: 01-01-2020

Accepted: 03-02-2020

\section{SS Wagh}

Assistant Professor, Plant

Pathology Section, College of

Agriculture, Nandurbar,

Maharashtra, India

AP Suryawanshi

Professor, Department of Plant Pathology, VNMKV, Parbhani, Maharashtra, India

\section{SL Badgujar}

Associate Professor, Department of Plant Pathology, VNMKV, Parbhani, Maharashtra, India

\section{Ambadkar}

Assistant Professor Department of Plant Pathology, VNMKV,

Parbhani, Maharashtra, India
Corresponding Author: SS Wagh

Assistant Professor, Plant Pathology Section, College of Agriculture, Nandurbar, Maharashtra, India

\section{Integrated management of Alternaria blight of safflower caused by Alternaria carthami under field conditions}

\author{
SS Wagh, AP Suryawanshi, SL Badgujar and CV Ambadkar
}

DOI: $\underline{\text { https://doi.org/10.22271/chemi.2020.v8.i2ad.9040 }}$

\begin{abstract}
Safflower (Carthamus tinctorius L.), is one of the most popular oilseeds crop grown throughout the world. The crop suffers many fungal diseases among that leaf spot/blight caused by Alternaria carthami (Chowdhury) has become one of the major constraints in the production and productivity of safflower all over the country as such and in the state of Maharashtra also. The disease has been reported to cause 25 to 60 per cent yield losses in safflower. Management of safflower Alternaria blight with chemicals alone needs to be discouraged due to their ill- effects. Therefore, present field experiments were conducted to manage the disease with integration of the effective fungicides, bioagents and phytoextracts under natural epi-phytotic condition at VNMKV, Parbhani, Maharashtra. The experiment was designed with RBD and all the treatments replicated thrice using Cv. Manjira, during Rabi, 2013 and 2014.

Results of the integrated management studies revealed that all treatments were found effective in controlling Alternaria blight disease. However, comparatively least average disease intensity (14.34\%) and its highest reduction $(74.36 \%$ ) were recorded with the combination seed treatment and foliar spray in treatment $\mathrm{T}_{9}$ of $T$. viride ST @ $10 \mathrm{~g} / \mathrm{kg}+$ Garlic clove extract ST @ $10 \mathrm{ml} / \mathrm{kg}+$ Hexaconazole FS @ $0.1 \%$,followed by treatment T4 of Hexaconazole ST @ 1 ml $/ \mathrm{kg}+$ It's FS @ $0.1 \%$ (Av. PDI : 15.46 \%, Av PDC : $72.37 \%$ ) and $\mathrm{T}_{10}$ of T. viride ST @ $1 \mathrm{ml} / \mathrm{kg}+$ Garlic clove extract ST @ $10 \mathrm{ml} / \mathrm{kg}+$ Mancozeb FS @ $0.25 \%$ (Av. PDI : $17.25 \%$, Av. PDC : $69.16 \%$ ). Significant increase in seed yield up to 54.56 per cent and highest ICBR (3.22) was recorded in treatment $\mathrm{T}_{9}$ (T. viride ST @ $10 \mathrm{~g} / \mathrm{kg}+$ Garlic clove extract ST@10 ml/kg + Hexaconazole FS @ $0.1 \%$ ).
\end{abstract}

Keywords: Alternaria carthami, safflower, intensity, management, field evaluation

\section{Introduction}

Safflower, (Carthamus tinctorius L.), is one of the world's oldest important oilseeds crop of the semi-arid regions belonging to the family Asteraceae (Compositae). The major safflowerproducing countries of the world are: India, Mexico, USA, Argentina, Canada and China. Safflower is also affected by many biotic and abiotic stresses. Of the biotic agents, fungi cause major diseases, followed by bacteria, viruses and nematodes (Bhale et al., 1998) ${ }^{[4]}$.

Among these diseases, at present leaf spot/blight caused by Alternaria carthami (Chowdhury) is widespread and have continued to be the major constraints in the production and productivity of safflower all over the country in general as well as in the state of Maharashtra particularly. The disease (A. carthami) has been reported to cause 25 to 60 per cent yield losses all over India (Singh and Prasad, 2005) ${ }^{[12]}$ and 20 to 80 per cent in Maharashtra state (Anonymous, 2010) ${ }^{[2]}$, along with drastic reduction in seed size, seed volume, seed test weight as well as per cent oil content.

Safflower cultivars/varieties presently under cultivation do not posses proven field resistance or tolerance and majority of them are more or less prone to the leaf spot/blight disease $(A$. carthami), under such circumstances fungicides provide the most reliable means of controlling foliage diseases. Present day public perceptions and environmental hazards are compelling to search for alternative eco-friendly disease management strategies, for which integration of various cultural, biological and chemical methods might be the solution (Jagan et al., 2013) ${ }^{[5]}$.

\section{Materials and Methods}

Integrated field evaluation of fungicides, bioagents and botanicals

The field experiments were conducted on the Research Farms of AICRP (Safflower) and 
Department of Agronomy, Vasantrao Naik Marathwada Krishi Vidyapeeth, Parbhani during Rabi, 2013-14 and 201415 , respectively to evaluate the efficacy of those fungicides, botanicals and bioagents which were found most effective against $A$. carthami, during present in vitro studies.

A total of three sprayings of all the treatments were undertaken at an interval of 15 days, starting first spraying at first appearance of Alternaria blight disease symptoms. One plot/treatment/replication was maintained as unsprayed control, without receiving any seed treatment or foliar sprays of the fungicides/bioagents/botanicals.
Ten plants per treatment per replication were selected randomly and tagged for recording observations on disease intensity. Three leaves (bottom, middle and top) from main branch on each observation plant were selected and foliage Alternaria blight disease intensity were recorded, applying 0-9 grade disease rating scale (Mayee and Datar, 1986) ${ }^{[8]}$, one day before each sprayings and last observation at 15 days after last spraying. Per cent disease intensity was calculated as given below.

\section{Treatments details}

\begin{tabular}{|c|c|c|}
\hline T. No. & Treatments & Rate (g or ml / kg seed / spray \%) \\
\hline $\mathrm{T}_{1}$ & Hexaconazole 5 EC (ST) & $1 \mathrm{ml} / \mathrm{kg}$ \\
\hline $\mathrm{T}_{2}$ & Mancozeb 75 WP (ST) & $2.5 \mathrm{~g} / \mathrm{kg}$ \\
\hline $\mathrm{T}_{3}$ & Mancozeb $63 \%$ + Carbendazim 12\% (SAAF 75 WP) ST & $2 \mathrm{~g} / \mathrm{kg}$ \\
\hline $\mathrm{T}_{4}$ & Hexaconazole 5 EC (ST) + It's FS & $1 \mathrm{ml} / \mathrm{kg}+0.1 \%$ \\
\hline $\mathrm{T}_{5}$ & Mancozeb 75 WP (ST) + It's FS & $2.5 \mathrm{~g} / \mathrm{kg}+0.25 \%$ \\
\hline $\mathrm{T}_{6}$ & SAAF 75 WP (ST) + It's FS & $2 \mathrm{~g} / \mathrm{kg}+0.2 \%$ \\
\hline $\mathrm{T}_{7}$ & Trichodermaviride $\left(2 \times 10^{7} \mathrm{cfu} / \mathrm{g}\right) \mathrm{ST}$ & $10 \mathrm{~g} / \mathrm{kg}$ \\
\hline $\mathrm{T}_{8}$ & Garlic clove extract @ 10\% (ST) & $10 \mathrm{ml} / \mathrm{kg}$ \\
\hline $\mathrm{T}_{9}$ & T. viride (ST) + Garlic clove extract @ $10 \%$ (ST) + Hexaconazole 5 EC (FS) & $10 \mathrm{~g} / \mathrm{kg}+10 \mathrm{ml} / \mathrm{kg}+0.1 \%$ \\
\hline $\mathrm{T}_{10}$ & T. viride (ST) + Garlic clove extract @ $10 \%(\mathrm{ST})+$ Mancozeb 75 WP (FS) & $10 \mathrm{~g} / \mathrm{kg}+10 \mathrm{ml} / \mathrm{kg}+0.25 \%$ \\
\hline $\mathrm{T}_{11}$ & T. viride (ST) + Garlic clove extract @ $10 \%(\mathrm{ST})+$ SAAF 75 WP (FS) & $10 \mathrm{~g} / \mathrm{kg}+10 \mathrm{ml} / \mathrm{kg}+0.2 \%$ \\
\hline $\mathrm{T}_{12}$ & Hexaconazole 5 EC (ST)+ SAAF 75 WP (FS) & $1 \mathrm{ml} / \mathrm{kg}+0.2 \%$ \\
\hline $\mathrm{T}_{13}$ & Mancozeb 75 WP (ST) + SAAF 75 WP (FS) & $2.5 \mathrm{~g} / \mathrm{kg}+0.2 \%$ \\
\hline $\mathrm{T}_{14}$ & Control (Untreated) & -- \\
\hline
\end{tabular}

$$
\mathrm{PDI}=\frac{\text { Summation of numerical ratings observed }}{\text { No. of leaves } / \text { plants observed } \mathrm{x} \text { maximum grade }} \mathrm{X} 100
$$

Further, per cent disease control (PDC) was worked out applying the following formula.

$$
\text { Per cent disease control (PDC) }=\frac{\text { PDI in control plot }-\mathrm{PDI} \text { in treatment plot }}{\mathrm{PDI} \text { in control plot }}
$$

\section{Seed yield data and ICBR}

In both the field experiments (Rabi, 2013-14 and 2014-15) after maturity plot harvested at maturity in all the treatments replicated and cumulative seed yield data was presented (q/ha).

To find out the most effective and economical treatment, the incremental cost: benefit ratio (ICBR) was worked out. For this the expenditure incurred on the inputs viz., fungicides, bioagents, botanicals and labour charges on spraying were taken into account.

\section{Pooled analysis}

The data obtained on per cent blight disease intensity, seed test weight and seed yield in both the experiments (Rabi, 2013-14 and 2014-15) were subjected to pooled analysis and interpreted the results thereof.

\section{Results and Discussion \\ Integrated efficacy of various treatments against safflower Alternaria blight}

A total 13 treatments, comprising three fungicides (systemic, non-systemic and combi fungicide one each), one fungal ( $T$. viride) bioagent and one phytoextract (A. sativum) found most effective against $A$. carthami under present in vitro evaluation were integrated (alone or in combination) as seed treatment and or foliar sprayings for the management of Alternaria blight disease in safflower (cv. Manjira) during Rabi, 2013-14 and 2014-15 seasons. The results obtained on pooled per cent disease intensity, pooled seed test weight, pooled seed yield and pooled incremental cost benefit ratio (ICBR) are

\begin{tabular}{|c|c|c|c|c|c|c|c|}
\hline \multirow[b]{2}{*}{$\begin{array}{l}\text { T. } \\
\text { No. }\end{array}$} & \multirow[b]{2}{*}{ Treatments } & \multicolumn{4}{|c|}{ Disease Intensity* (\%) } & \multirow{2}{*}{$\begin{array}{c}\text { Av. PDI } \\
(\%)\end{array}$} & \multirow{2}{*}{$\begin{array}{c}\text { Av. } \\
\text { PDC } \\
(\%)\end{array}$} \\
\hline & & $\begin{array}{c}\text { At } 1^{\text {st }} \\
\text { Appear }\end{array}$ & $\begin{array}{l}\text { After } \mathbf{I}^{\text {st }} \\
\text { Spray }\end{array}$ & $\begin{array}{l}\text { After II }{ }^{\text {nd }} \\
\text { Spray }\end{array}$ & $\begin{array}{l}15 \text { days After } \\
\text { III' }{ }^{\text {rd }} \text { Spray }\end{array}$ & & \\
\hline $\mathrm{T}_{1}$ & Hexaconazole 5 EC ST @ 1 ml/kg & $\begin{array}{c}19.50 \\
(10.19)\end{array}$ & $\begin{array}{c}27.58 \\
(17.26)\end{array}$ & $\begin{array}{c}32.04 \\
(19.44)\end{array}$ & $23.31(12.44)$ & $\begin{array}{c}25.61 \\
(14.83)\end{array}$ & 54.24 \\
\hline $\mathrm{T}_{2}$ & Mancozeb 75 WP ST @ $2.5 \mathrm{~g} / \mathrm{kg}$ & $\begin{array}{c}19.96 \\
(11.51)\end{array}$ & $\begin{array}{c}31.46 \\
(18.33)\end{array}$ & $\begin{array}{c}34.83 \\
(20.22)\end{array}$ & $24.52(14.22)$ & $\begin{array}{c}27.69 \\
(16.07)\end{array}$ & 50.51 \\
\hline $\mathrm{T}_{3}$ & Mancozeb 63\% + Carbendazim 12\% (SAAF) ST @ 2 g/kg & $\begin{array}{c}22.27 \\
(12.87) \\
\end{array}$ & $\begin{array}{c}32.90 \\
(19.21) \\
\end{array}$ & $\begin{array}{c}35.56 \\
(20.67) \\
\end{array}$ & $26.83(15.59)$ & $\begin{array}{c}29.39 \\
(17.09) \\
\end{array}$ & 47.48 \\
\hline $\mathrm{T}_{4}$ & Hexaconazole 5 EC ST @ 1 ml/kg + It’s FS @0.1% & $\begin{array}{c}11.87 \\
(06.82)\end{array}$ & $\begin{array}{l}16.74 \\
(9.63)\end{array}$ & $\begin{array}{c}21.07 \\
(11.99)\end{array}$ & $12.16(6.99)$ & $\begin{array}{l}15.46 \\
(8.86)\end{array}$ & 72.37 \\
\hline $\mathrm{T}_{5}$ & Mancozeb ST@ 2.5 g/kg + It’s FS@0.25\% & $\begin{array}{l}16.12 \\
(9.27)\end{array}$ & $\begin{array}{c}22.46 \\
(12.98)\end{array}$ & $\begin{array}{c}26.87 \\
(15.42)\end{array}$ & $15.21(8.76)$ & $\begin{array}{c}20.16 \\
(11.61)\end{array}$ & 63.97 \\
\hline
\end{tabular}
presented in the Tables 1 to 3 and Fig 1 and 2 .

Table 1: Effect of various treatments integration on pooled mean Alternaria blight intensity in safflower at various intervals (Rabi, 2013-14 and 2014-15) 


\begin{tabular}{|c|c|c|c|c|c|c|c|}
\hline $\mathrm{T}_{6}$ & SAAF ST@2 g/kg + It’s FS@0.2% & $\begin{array}{c}17.69 \\
(11.05)\end{array}$ & $\begin{array}{c}25.60 \\
(15.51)\end{array}$ & $\begin{array}{c}30.67 \\
(18.21)\end{array}$ & $16.39(10.38)$ & $\begin{array}{c}22.59 \\
(13.79)\end{array}$ & 59.63 \\
\hline $\mathrm{T}_{7}$ & T.viride $\left(2 \mathrm{X} 10^{7} \mathrm{cfu} / \mathrm{g}\right) \mathrm{ST} @ 10 \mathrm{~g} / \mathrm{kg}$ & $\begin{array}{c}25.92 \\
(15.08) \\
\end{array}$ & $\begin{array}{c}37.42 \\
(22.04) \\
\end{array}$ & $\begin{array}{c}42.75 \\
(25.15) \\
\end{array}$ & $30.48(17.78)$ & $\begin{array}{c}34.14 \\
(20.01) \\
\end{array}$ & 38.98 \\
\hline $\mathrm{T}_{8}$ & Garlic clove extract $10 \% \mathrm{ST} @ 10 \mathrm{ml} / \mathrm{kg}$ & $\begin{array}{c}29.87 \\
(17.38)\end{array}$ & $\begin{array}{l}41.37 \\
(24.54)\end{array}$ & $\begin{array}{c}46.70 \\
(27.84)\end{array}$ & $34.43(20.19)$ & $\begin{array}{l}38.09 \\
(22.49)\end{array}$ & 31.92 \\
\hline $\mathrm{T}_{9}$ & $\begin{array}{l}\text { T. viride ST @ } 10 \mathrm{~g} / \mathrm{kg}+\text { Garlic clove extract (10\%) ST @ } 10 \\
\text { ml } / \mathrm{kg}+\text { Hexaconazole 5 EC FS @ 0.1\% }\end{array}$ & $\begin{array}{l}13.43 \\
(7.72)\end{array}$ & $\begin{array}{l}13.67 \\
(7.86)\end{array}$ & $\begin{array}{c}18.99 \\
(10.95)\end{array}$ & $11.30(6.49)$ & $\begin{array}{l}14.34 \\
(8.26)\end{array}$ & 74.36 \\
\hline $\mathrm{T}_{10}$ & $\begin{array}{c}\text { T. viride ST @ } 1 \mathrm{ml} / \mathrm{kg}+\text { Garlic clove extract (10\%) ST @ } 10 \\
\text { ml } / \mathrm{kg}+\text { Mancozeb 75 WP FS @ 0.25\% }\end{array}$ & $\begin{array}{l}13.56 \\
(7.79) \\
\end{array}$ & $\begin{array}{c}19.66 \\
(11.34) \\
\end{array}$ & $\begin{array}{c}23.76 \\
(13.74) \\
\end{array}$ & $12.05(6.93)$ & $\begin{array}{l}17.25 \\
(9.95)\end{array}$ & 69.16 \\
\hline $\mathrm{T}_{11}$ & $\begin{array}{l}\text { T. viride ST @ } 1 \mathrm{ml} / \mathrm{kg}+\text { Garlic clove extract (10 \%) ST @ } 10 \\
\text { ml } / \mathrm{kg}+\text { SAAF 75 WP FS @ 0.2\% }\end{array}$ & $\begin{array}{c}17.69 \\
(11.05)\end{array}$ & $\begin{array}{c}25.60 \\
(15.51) \\
\end{array}$ & $\begin{array}{c}30.67 \\
(18.21) \\
\end{array}$ & $19.50(10.31)$ & $\begin{array}{c}23.36 \\
(13.77)\end{array}$ & 58.25 \\
\hline $\mathrm{T}_{12}$ & Hexaconazole ST @ 1 ml/kg + SAAF 75 WP FS @ $0.2 \%$ & $\begin{array}{c}20.68 \\
(12.30) \\
\end{array}$ & $\begin{array}{c}26.03 \\
(14.77) \\
\end{array}$ & $\begin{array}{c}31.03 \\
(17.78) \\
\end{array}$ & $20.62(12.29)$ & $\begin{array}{c}24.59 \\
(14.29) \\
\end{array}$ & 56.05 \\
\hline $\mathrm{T}_{13}$ & Mancozeb 75 WP ST @ 2.5 g/kg + SAAF FS @ $0.2 \%$ & $\begin{array}{c}18.87 \\
(11.56)\end{array}$ & $\begin{array}{c}28.12 \\
(15.41)\end{array}$ & $\begin{array}{c}32.54 \\
(18.38)\end{array}$ & $19.57(11.99)$ & $\begin{array}{c}24.77 \\
(14.34)\end{array}$ & 55.72 \\
\hline $\mathrm{T}_{14}$ & Control (Untreated) & $\begin{array}{c}30.08 \\
(17.52)\end{array}$ & $\begin{array}{c}43.67 \\
(25.99)\end{array}$ & $\begin{array}{c}64.72 \\
(40.34) \\
\end{array}$ & $85.34(59.50)$ & $\begin{array}{c}55.95 \\
(35.84)\end{array}$ & -- \\
\hline-- & S.E. \pm & 0.95 & 1.59 & 1.60 & 1.67 & -- & -- \\
\hline-- & C.D. $(\mathrm{P}=0.05)$ & 2.77 & 4.61 & 4.66 & 4.84 & -- & -- \\
\hline-- & $\mathrm{CV}$ & 15.44 & 16.82 & 16.02 & 17.93 & -- & -- \\
\hline
\end{tabular}

*: Mean of two replications, Figures in parentheses are arcsine transformed values, PDI: Per cent disease intensity, PDC : Percent disease control, ST : Seed treatment, FS: Foliar spray

Table 2: Effect of various treatments integration on pooled mean test weight and seed yield in safflower (Rabi, 2013-14 and 2014-15)

\begin{tabular}{|c|c|c|c|c|c|}
\hline \begin{tabular}{|c|} 
Tr. \\
No.
\end{tabular} & Treatments & $\begin{array}{c}\text { Mean } \\
(\%)\end{array}$ & $\begin{array}{c}\text { Test weight } \\
\text { (g) }\end{array}$ & $\begin{array}{l}\text { Seed Yield } \\
(\mathbf{q} / \mathrm{ha})\end{array}$ & $\begin{array}{l}\text { \% Yield Increase over } \\
\text { control }\end{array}$ \\
\hline $\mathrm{T}_{1}$ & Hexaconazole 5 EC ST @ 1 ml/kg & $\begin{array}{c}25.61 \\
(14.83)\end{array}$ & 3.65 & 8.92 & 26.23 \\
\hline $\mathrm{T}_{2}$ & Mancozeb 75 WP ST @ $2.5 \mathrm{~g} / \mathrm{kg}$ & $\begin{array}{c}27.69 \\
(16.07)\end{array}$ & 3.52 & 8.50 & 22.59 \\
\hline $\mathrm{T}_{3}$ & Mancozeb 63\% + Carbendazim 12\% (SAAF) ST @ 2 g/kg & $\begin{array}{c}29.39 \\
(17.09)\end{array}$ & 3.38 & 8.14 & 19.16 \\
\hline $\mathrm{T}_{4}$ & Hexaconazole 5 EC ST @ 1 ml/kg + It’s FS @ $0.1 \%$ & $\begin{array}{l}15.46 \\
(8.86) \\
\end{array}$ & 4.94 & 13.63 & 51.72 \\
\hline $\mathrm{T}_{5}$ & Mancozeb ST @ 2.5 g/kg + It’s FS@0.25\% & $\begin{array}{c}20.16 \\
(11.61)\end{array}$ & 4.45 & 12.38 & 46.85 \\
\hline $\mathrm{T}_{6}$ & $\begin{array}{c}\text { SAAF ST @ } 2 \mathrm{~g} / \mathrm{kg}+\mathrm{It} \text { 's FS } \\
\text { @ } 0.2 \%\end{array}$ & $\begin{array}{c}22.59 \\
(13.79)\end{array}$ & 4.30 & 10.73 & 38.68 \\
\hline $\mathrm{T}_{7}$ & T.viride $\left(2 \mathrm{X} 10^{7} \mathrm{cfu} / \mathrm{g}\right) \mathrm{ST} @ 10 \mathrm{~g} / \mathrm{kg}$ & $\begin{array}{c}34.14 \\
(20.01) \\
\end{array}$ & 3.23 & 7.93 & 17.02 \\
\hline $\mathrm{T}_{8}$ & Garlic clove extract $10 \%$ ST @ 10 ml $/ \mathrm{kg}$ & $\begin{array}{c}38.09 \\
(22.49)\end{array}$ & 3.17 & 7.60 & 13.42 \\
\hline $\mathrm{T}_{9}$ & $\begin{array}{c}\text { T. viride ST @ } 10 \mathrm{~g} / \mathrm{kg}+\text { Garlic clove extract (10\%) ST @ } 10 \mathrm{ml} / \mathrm{kg}+ \\
\text { Hexaconazole 5 EC FS @ 0.1\% }\end{array}$ & $\begin{array}{l}14.34 \\
(8.26)\end{array}$ & 5.10 & 14.48 & 54.56 \\
\hline $\mathrm{T}_{10}$ & $\begin{array}{c}\text { T. viride ST @ } 1 \text { ml } / \mathrm{kg}+\text { Garlic clove extract (10\%) ST @ } 10 \mathrm{ml} / \mathrm{kg}+ \\
\text { Mancozeb 75 WP FS @ 0.25\% }\end{array}$ & $\begin{array}{l}17.25 \\
(9.95)\end{array}$ & 4.67 & 13.02 & 49.46 \\
\hline $\mathrm{T}_{11}$ & $\begin{array}{c}\text { T. viride ST @ } 1 \mathrm{ml} / \mathrm{kg}+\text { Garlic clove extract }(10 \%) \text { ST @ } 10 \mathrm{ml} / \mathrm{kg}+ \\
\text { SAAF 75 WP FS @ } 0.2 \%\end{array}$ & $\begin{array}{c}23.36 \\
(13.77) \\
\end{array}$ & 4.14 & 10.45 & 37.03 \\
\hline $\mathrm{T}_{12}$ & Hexaconazole ST @ 1 ml/kg + SAAF 75 WP FS @ $0.2 \%$ & $\begin{array}{c}24.59 \\
(14.29) \\
\end{array}$ & 4.00 & 10.10 & 34.85 \\
\hline $\mathrm{T}_{13}$ & Mancozeb 75 WP ST @ 2.5 g/kg + SAAF FS @ $0.2 \%$ & $\begin{array}{c}24.77 \\
(14.34)\end{array}$ & 3.74 & 9.47 & 30.52 \\
\hline $\mathrm{T}_{14}$ & Control (Untreated) & $\begin{array}{c}55.95 \\
(35.84) \\
\end{array}$ & 2.79 & 6.58 & -- \\
\hline \begin{tabular}{|l|l}
-- \\
\end{tabular} & S.E. \pm & -- & 0.08 & 0.64 & -- \\
\hline \begin{tabular}{|l|}
-- \\
\end{tabular} & C.D. $(\mathrm{P}=0.05)$ & -- & 0.23 & 1.86 & -- \\
\hline \begin{tabular}{|l}
-- \\
\end{tabular} & $\mathrm{CV}$ & -- & 5.44 & 9.48 & -- \\
\hline
\end{tabular}

*: Mean of two replications, Conc.: Concentration, ST: Seed treatment, Figures in parentheses are arc sine transformed values, FS: Foliar spray.

Table 3: Pooled mean of incremental cost: benefit ratio (ICBR) (2013-14 and 2014 -15)

\begin{tabular}{|c|c|c|c|c|}
\hline Tr. No. & Treatments & \multicolumn{2}{|c|}{\begin{tabular}{|c|} 
ICBR \\
$2013-14 \mid 2014-15$ \\
\end{tabular}} & Pooled Mean \\
\hline $\mathrm{T}_{1}$ & Hexaconazole 5EC ST @ 1 ml/kg & 2.23 & 2.06 & 2.15 \\
\hline$T_{2}$ & Mancozeb 75 WP ST @ $2.5 \mathrm{~g} / \mathrm{kg}$ & 2.13 & 1.96 & 2.05 \\
\hline $\mathrm{T}_{3}$ & Mancozeb 63\% + Carbendazim 12\% (SAAF) ST @ 2 g/kg & 2.05 & 1.86 & 1.96 \\
\hline $\mathrm{T}_{4}$ & Hexaconazole 5EC ST @ 1 ml/kg + It's FS @ $0.1 \%$ & 3.04 & 2.99 & 3.02 \\
\hline $\mathrm{T}_{5}$ & Mancozeb ST @ $2.5 \mathrm{~g} / \mathrm{kg}$ + It's FS @ $0.25 \%$ & 2.88 & 2.81 & 2.85 \\
\hline $\mathrm{T}_{6}$ & SAAF ST @ 2 g/kg + It's FS @ $0.2 \%$ & 2.43 & 2.32 & 2.38 \\
\hline
\end{tabular}




\begin{tabular}{|c|c|c|c|c|}
\hline $\mathrm{T}_{7}$ & T.viride $\left(2 \mathrm{X} 10^{7} \mathrm{cfu} / \mathrm{g}\right) \mathrm{ST} @ 10 \mathrm{~g} / \mathrm{kg}$ & 2.00 & 1.81 & 1.91 \\
\hline $\mathrm{T}_{8}$ & Garlic clove extract $10 \%$ ST @ $10 \mathrm{ml} / \mathrm{kg}$ & 1.93 & 1.73 & 1.83 \\
\hline $\mathrm{T}_{9}$ & T. viride ST @ 10 g/kg + Garlic clove extract (10\%) ST @ 10 ml $/ \mathrm{kg}+$ Hexaconazole 5 EC FS @ $0.1 \%$ & 3.33 & 3.31 & 3.32 \\
\hline $\mathrm{T}_{10}$ & T. viride ST @ 1 ml/kg + Garlic clove extract (10\%) ST @ 10 ml/kg + Mancozeb 75 WP FS @ $0.25 \%$ & 2.90 & 2.84 & 2.87 \\
\hline $\mathrm{T}_{11}$ & T. viride ST @ 1 ml/kg + Garlic clove extract (10\%) ST @ 10 ml/kg + SAAF 75 WP FS @ 0.2\% & 2.36 & 2.24 & 2.30 \\
\hline $\mathrm{T}_{12}$ & Hexaconazole ST @ 1 ml/kg + SAAF 75 WP FS @ $0.2 \%$ & 2.16 & 2.16 & 2.16 \\
\hline $\mathrm{T}_{13}$ & Mancozeb 75 WP ST @ 2.5 g/kg + SAAF FS @ $0.2 \%$ & 2.29 & 2.01 & 2.15 \\
\hline $\mathrm{T}_{14}$ & Control (Untreated) & 1.71 & 1.48 & 1.60 \\
\hline
\end{tabular}

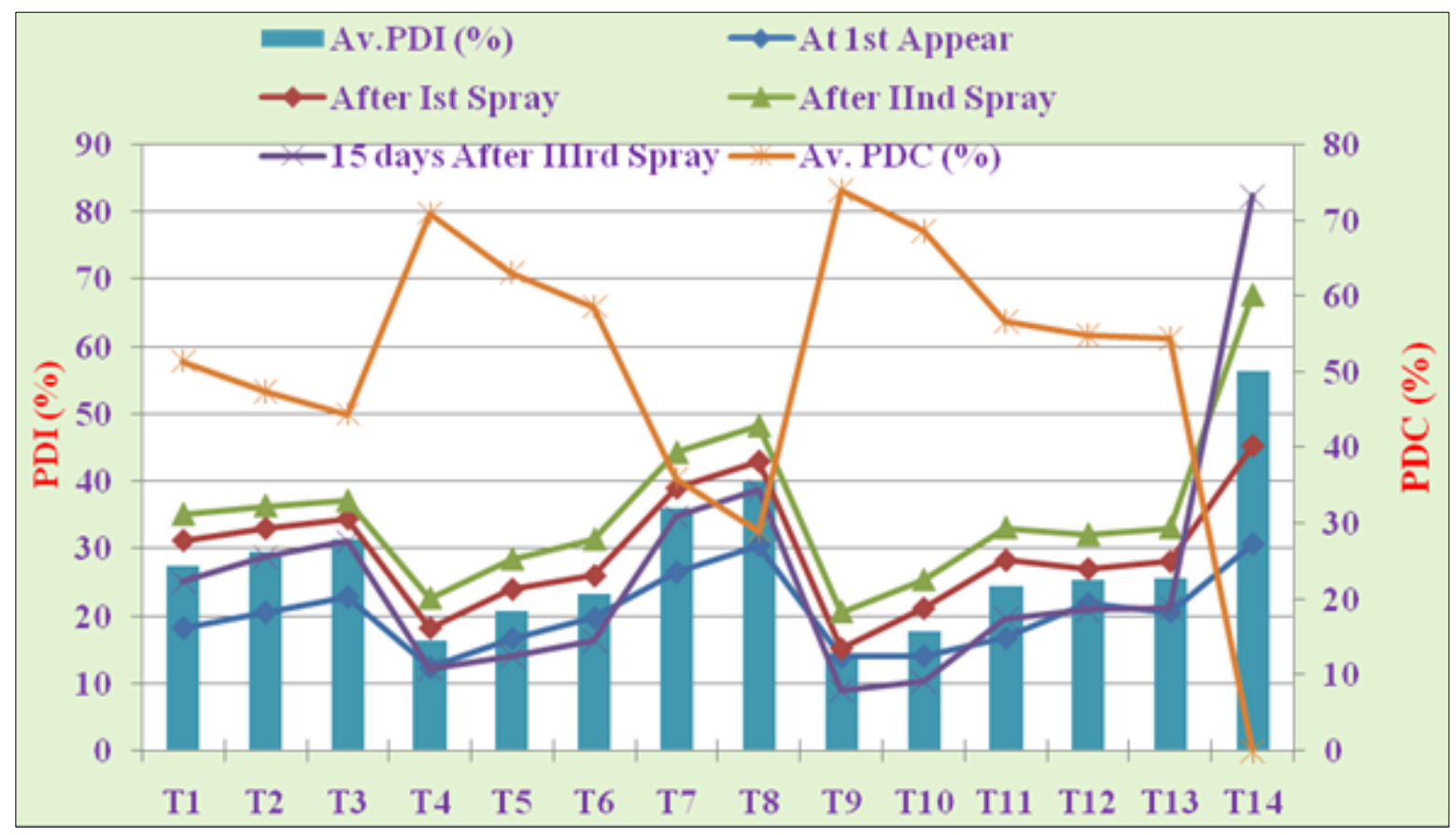

Fig. 1: Integrated efficacy of various treatments against safflower Alternaria blight disease during Rabi, 2013-14

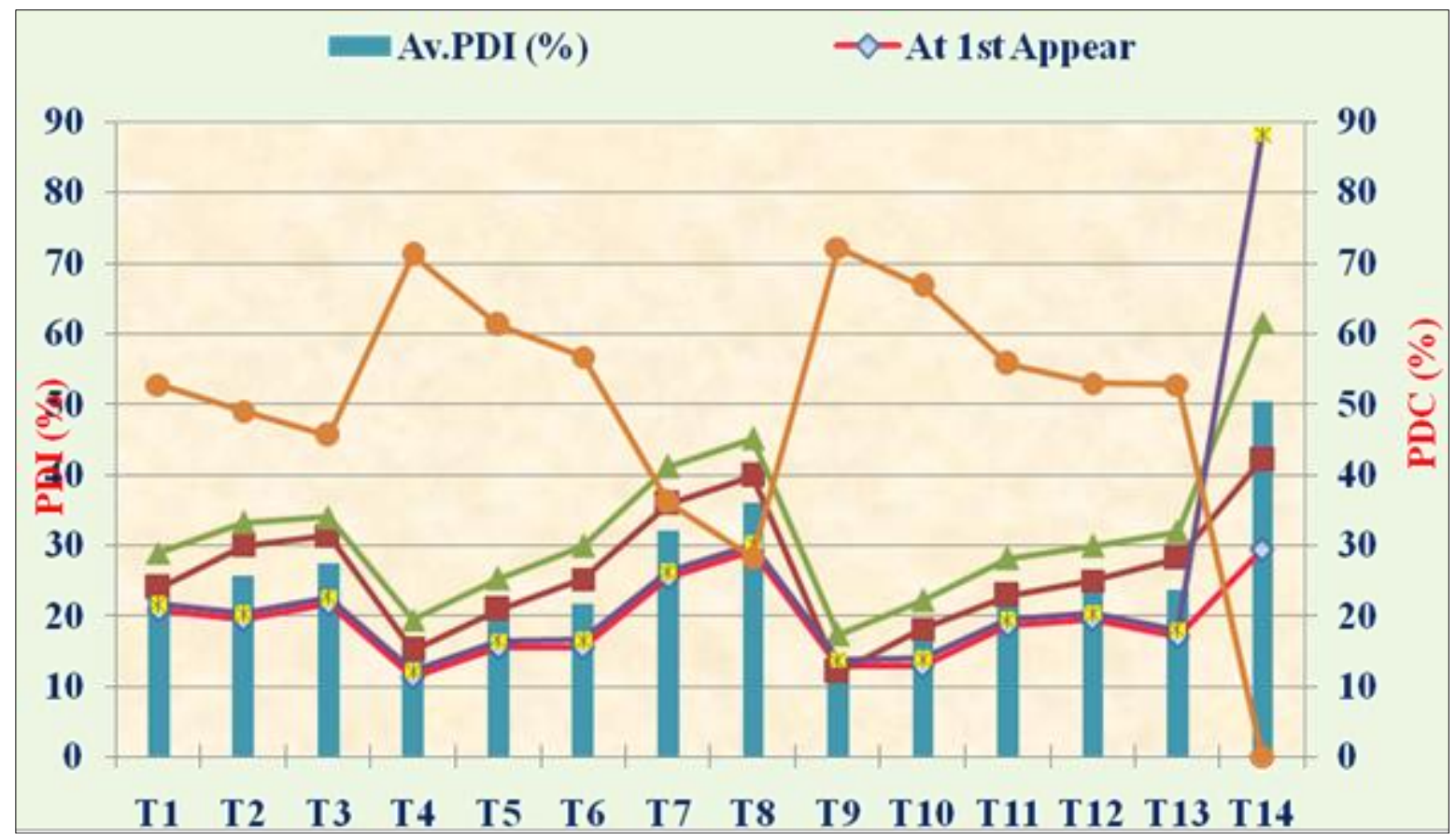

Fig. 2: Integrated efficacy of various treatments against safflower Alternaria blight intensity disease during Rabi, 2014-15

\section{Pooled mean results (Rabi, 2013-14 and 2014-15) \\ Percent disease control}

The pooled results (Table 1 ) revealed that all the treatments (ST and FS) significantly influenced Alternaria blight mean disease intensity recorded at various intervals in safflower (cv. Manjira). The disease was found to appear first around 25 to 30 days and its mean intensity at first appearance was ranged from $11.87\left(\mathrm{~T}_{4}\right)$ to $29.87\left(\mathrm{~T}_{8}\right)$ per cent, as against
30.08 per cent in untreated control; further, it was found to be increased steadily upto second spraying and decreased thereafter third spray treatment.

Average percent disease control recorded with all the treatments was ranged from $74.36\left(\mathrm{~T}_{9}\right)$ to $31.92\left(\mathrm{~T}_{8}\right)$ per cent; However, comparatively least average disease intensity (14.34 $\%)$ and its highest reduction $(74.36 \%)$ were recorded with the treatment $\mathrm{T}_{9}$ of $T$. viride ST @ $10 \mathrm{~g} / \mathrm{kg}+$ Garlic clove extract 
ST @ 10 ml/kg + Hexaconazole FS @ $0.1 \%$. The second and third best treatment found were $\mathrm{T}_{4}$ of Hexaconazole ST @ 1 ml/kg + It's FS@0.1\%(Av. PDI : 15.46\%, Av PDC : 72.37 $\%$ ) and $\mathrm{T}_{10}$ of $T$. viride ST @ $1 \mathrm{ml} / \mathrm{kg}+$ Garlic clove extract ST @ 10 ml $/ \mathrm{kg}+$ Mancozeb FS @ $0.25 \%$ (Av. PDI : 17.25 $\%$, Av. PDC : $69.16 \%)$. These were followed by the treatment $\mathrm{T}_{10}$ of $T$. viride $\mathrm{ST} @ 1 \mathrm{ml} / \mathrm{kg}+$ Garlic clove extract ST @ 10 ml $/ \mathrm{kg}+$ Mancozeb FS @ $0.25 \%$ (Av. PDI : 17.25 $\%$, Av. PDC : 69.16\%), T5 of Mancozeb ST @ $2.5 \mathrm{~g} / \mathrm{kg}+\mathrm{It}$ 's FS@0.25\% (Av. PDI : 20.16\%, Av. PDC : $63.97 \%$ ), $\mathrm{T}_{11}$ of T. viride ST @ $1 \mathrm{ml} / \mathrm{kg}+$ Garlic clove extract ST @ $10 \mathrm{ml} / \mathrm{kg}$ + SAAF FS @ $0.2 \%$ (Av. PDI : 23.36 \%, Av PDC : 58.25 $\%)$. Rest of the treatments recorded average disease intensity in the range of $24.59\left(\mathrm{~T}_{12}\right)$ to $38.09\left(\mathrm{~T}_{7}\right)$ per cent and average disease reduction / control in the range of $31.92\left(\mathrm{~T}_{8}\right)$ to 56.05 $\left(\mathrm{T}_{12}\right)$ per cent.

\section{Effect on mean test weight and seed yield}

The pooled results (Table 2) indicated that pooled mean seeds test weight $(\mathrm{g})$ and seed yield $(\mathrm{q} / \mathrm{h})$ were significantly influenced with various treatments imposed to manage Alternaria blight disease in safflower crop, which were ranged from 3.17 to $5.10 \mathrm{~g}$ and 7.60 to $14.48 \mathrm{q} / \mathrm{ha}$, respectively, against least mean test weight $(2.79 \mathrm{~g})$ and seed yield $(6.58$ $\mathrm{q} / \mathrm{ha}$ ) in untreated control. The per cent increase in seed yield with various treatments, over untreated control was ranged from 13.42 to 54.56 per cent. Among the treatments, significantly highest mean test weight $(5.10 \mathrm{~g})$, seed yield (14.48 q/ha) and increase in seed yield $(54.56 \%)$ with least mean disease intensity $(14.34 \%)$ were recorded with the treatment $\mathrm{T}_{9}$ of $T$. viride $\mathrm{ST} @ 10 \mathrm{~g} / \mathrm{kg}+$ Garlic clove extract ST@ $10 \mathrm{ml} / \mathrm{kg}+$ Hexaconazole FS @ $0.1 \%$, followed by the treatment $\mathrm{T}_{4}$ of Hexaconazole ST@1 ml $/ \mathrm{kg}+$ It's FS@0.1 $\%$ (4.94 g, 13.63 q/ha and $51.72 \%), \mathrm{T}_{10}$ of T. viride ST @ 1 $\mathrm{ml} / \mathrm{kg}+$ Garlic clove extract ST @ $10 \mathrm{ml} / \mathrm{kg}+$ Mancozeb FS @ $0.25 \%$ (4.67 g, $13.02 \mathrm{q} / \mathrm{ha}$ and $49.46 \%$ ) and $\mathrm{T}_{5}$ of Mancozeb ST@2.5 g/kg+It's FS@0.25\% (4.45 g, 12.38 q/ha and $46.85 \%$ ),respectively with test weight, seed yield and per cent increase in seed yield, over untreated control. Rest of the treatments compared to untreated control recorded also better weight in the range of $3.17\left(\mathrm{~T}_{8}\right)$ to $4.30\left(\mathrm{~T}_{6}\right)$, seed yield in the range of $7.60 \mathrm{q} / \mathrm{ha}\left(\mathrm{T}_{8}\right)$ to $10.73 \mathrm{q} / \mathrm{ha}\left(\mathrm{T}_{6}\right)$ and increase in seed yield in the range of $13.42\left(\mathrm{~T}_{8}\right)$ to $38.68\left(\mathrm{~T}_{6}\right)$ per cent.

\section{Pooled mean of incremental cost : benefit ratio (ICBR)}

Results (Table 3) indicated that on the basis of two years (2013-14 and 2014-15) pooled mean data, the most economical treatment with highest mean ICBR (3.32) was $\mathrm{T}_{9}$ (T. viride ST @ $10 \mathrm{~g} / \mathrm{kg}+$ Garlic clove extract ST @ $10 \mathrm{ml} / \mathrm{kg}$ + Hexaconazole FS @ $0.1 \%$ ), followed by the treatments $\mathrm{T}_{4}$ (Hexaconazole ST @ 1 ml/kg + It's FS@0.1\%), T 10 . viride ST @ $1 \mathrm{ml} / \mathrm{kg}+$ Garlic clove extract ST @ $10 \mathrm{ml} / \mathrm{kg}+$ Mancozeb FS @ $0.25 \%$ ), T5 (Mancozeb ST @ $2.5 \mathrm{~g} / \mathrm{kg}+\mathrm{It}$ 's FS@0.25\%), T6 (SAAF ST@2 g/kg+ It's FS@0.2\%) and $\mathrm{T}_{11}$ (T. viride ST @ $1 \mathrm{ml} / \mathrm{kg}+$ Garlic clove extract ST @ $10 \mathrm{ml} / \mathrm{kg}$ + SAAF FS @ $0.2 \%$ ), with the ICBR, respectively of 3.02, 2.87, 2.85, 2.38 and 2.30, respectively.

These results are in conformity with the earlier findings of those workers who reported fungicides (systemic, nonsystemic and combi- fungicide), plant extracts / botanicals, bioagent along with or in combination ST or FS viz., (T. viride ST @ $10 \mathrm{~g} / \mathrm{kg}+$ Garlic clove extract ST @ $10 \mathrm{ml} / \mathrm{kg}+$ Hexaconazole FS@0.1\%), (Hexaconazole ST @ $1 \mathrm{ml} / \mathrm{kg}+$ It's FS@0.1\%), (T. viride ST @ 1 ml/kg + Garlic clove extract ST @ $10 \mathrm{ml} / \mathrm{kg}$ + Mancozeb FS @ $0.25 \%$ ), (Mancozeb ST@2.5 g/kg+It's FS@0.25\%), (SAAF ST@ $2 \mathrm{~g} / \mathrm{kg}+\mathrm{It}$ 's FS@0.2\%) and (T. viride ST @ $1 \mathrm{ml} / \mathrm{kg}+$ Garlic clove extract ST@10 ml/kg + SAAF FS @ $0.2 \%$ ) at various concentrations had significantly highest percent disease control, seed yield and ICBR ratio of $A$. carthami infecting safflower. Anonymous (2009) ${ }^{[1]}$ reported for the management of safflower Alternaria leaf spot disease that combi-fungicide Carbendazim 12\% + Mancozeb 63\% (SAAF $75 \mathrm{WP}) @ 0.2 \%$ recorded significantly least disease intensity (26.20\%), highest disease control $(70.70 \%)$ and highest seed yield (1036 kg/ha), followed by Carbendazim @ 0.1\% (36.50 $\%, 59.10 \%$ and $936 \mathrm{~kg} / \mathrm{ha})$ and Mancozeb @ $0.25 \%$ (42.20 $\%, 52.70 \%$ and $879 \mathrm{~kg} / \mathrm{ha}$ ) of disease intensity, disease control and seed yield, respectively. However, highest B: C ratio was reported with Carbendazim (14.5), followed by Mancozeb (10.3) and SAAF @ $0.2 \%$ (9.2). Basavarajappa et al. (2012) [3] reported under field conditions for the management of sunflower leaf spot (A. carthami) disease, Quintal $(0.2 \%)$ recorded least mean disease intensity (19.60 $\%)$ with highest seed yield (1593 kg/ha), followed by Difenconazole @ $0.05 \%$ (29.90\% and 1291 kg/ha) Mancozeb @ $0.05 \%$ (36.80\% and $1168 \mathrm{~kg} / \mathrm{ha})$, Chlorothalonil @ $0.2 \%$ (40.70\% and $1209 \mathrm{~kg} / \mathrm{ha})$, Hexaconazole @ $0.1 \%$ (43.10\% and $1210 \mathrm{~kg} / \mathrm{ha})$ and SAAF @ $0.2(43.30 \%$ and $1161 \mathrm{~kg} / \mathrm{ha})$, as against highest disease intensity $(87.90 \%)$ in control. On the basis of $\mathrm{B}: \mathrm{C}$ ratio the most economical treatments was Quintal (3.67), followed by Difenconazole (3.23) and Hexaconazole (3.19). Pawar et al. (2012) ${ }^{[11]}$ reported that Propiconazole gave significantly least mean disease severity $(27.40 \%)$ and maximum disease control (64.73\%) with highest seed yield $(1522 \mathrm{~kg} / \mathrm{ha})$ and maximum B:C ratio (2.27) against Alternaria carthami of safflower, followed by Mancozeb $(33.70 \%, 56.62,1413 \mathrm{~kg} / \mathrm{ha}$ and 2.16) and SAAF $(35.20 \%, 54.69 \%, 1388 \mathrm{~kg} / \mathrm{ha}$ and 2.05$)$ of the disease severity, disease control, seed yield and B:C ratio, respectively.

These results are in conformity with the findings of those reported earlier by several workers against, Alternaria sesame infecting sesame (Jeyalakshmi and Rettinassababady, 2009) [6], A. helianthi infecting sunflower (Karuna et al., 2012; Venkataramanamma et al., 2014) ${ }^{[7,13]}$.

Results of the present study obtained on integrated management of safflower Alternaria blight (A. carthami) disease with the fungicides viz., Hexaconazole, Mancozeb and SAAF, bioagents $T$. viride and botanical A. sativum which efficiently managed the disease with significant increase in seed yield and better ICBR are on the same line with the findings of those reported earlier by several workers. (Murumkar et al., 2009; Mesta et al., 2011) ${ }^{[10,9] .}$

Thus, for effective and economical management of safflower Alternaria blight (A. carthami) disease during Rabi, 2013-14 and 2014-15seasons, the integration of fungicides viz., Hexaconazole, Mancozeb and SAAF, bioagents T. viride and botanical A. sativum could be exploited on large scale under field conditions.

\section{References}

1. Anonymous. Safflower Annual Progress Report. Directorate of Oilseeds Research, Rajendranagar, Hyderabad, 2009, 111-131p.

2. Anonymous. Safflower Annual Progress Report. Directorate of Oilseeds Research, Rajendranagar, Hyderabad, 2010, 106-124p. 
3. Basavarajappa MP, Somanagoud G, Kambrekar DN, Hanumantharaya L. In vivo evaluation of new fungicide molecules for the management of leaf spot in safflower caused by Alternaria carthami Chowdhury. J Oilseeds Res. 2012; 29:346-348.

4. Bhale MS, Bhale U, Khare MN. Disease of important oilseed crops and their management. In pathological problems of economic crop plants and their management. (Eds. S.M. Paul Khurana), Scientific Publishers, Jodhpur, 1998, 251-279p.

5. Jagana M, Zacharia S, Lal AA, Basayya. Management of Alternaria blight in Mustard. Ann. Pl. Prot. Sci. 2013; 21(2):441-442.

6. Jeyalakshmi C, Rettinassababady C. Management of sesame diseases through botanicals and biocontrol agents. $5^{\text {th }}$ International Conference on Plant Pathology in the Globalized Era, Nov. 10-13, 2009, New Delhi, India, 2009, 323p.

7. Karuna K, Jagadish KS, Geetha KN, Shadakshari YG. Evaluation of efficacy of chemical fungicides and a plant products for the management of Alternaria blight of sunflower, Indian Phytopath. 2012; 65(3):305-306.

8. Mayee CD, Datar VV. Phytopathomethory: Technical Bulletin Published by Marathwada Agric. Univ., Parbhani (M.S.) India, 1986, 100-104p.

9. Mesta RK, Benagi VI, Kulkarni S, Basavarajappa MP. Management of Alternaria blight of sunflower through fungicides. Karnataka J Agric. Sci. 2011; 24(2):149-152.

10. Murumkar DR, Indi DV, Gud MA, Shinde SK, Deshpande AN. Fungicidal management of leaf spot of safflower caused by Alternaria carthami. J Maha. Agric. Univ. 2009; 34(1):54-56.

11. Pawar SV, Zote AK, Navgire KD, Mehetre SP, Patil CB. Management of Alternaria leaf spot in safflower using fungicides. J. Oilseeds Res. 2012; 29(Spl. Issue):368-370.

12. Singh V, Prasad RD. Integrated management of pests and diseases in safflower. Directorate of Oilseeds Research, Hyderabad, India, 2005, 49p.

13. Venkataramanamma K, Madhusudhan $P$, Neelima $S$, Narasimhudu Y. Field evaluation of fungicides for the management of Alternaria leaf blight of sunflower. Indian J Pl. Prot. 2014; 42(2):165-168. 